\title{
Photoconductive Effect of Liquid and Utilization of Solar Energy
}

\author{
Ziwei Chen ${ }^{1,2,3}$, Shihao Chen ${ }^{4}$ \\ ${ }^{1}$ The Key Laboratory of Electromagnetic Radiation and Detection Technology, Institute of Electronics, \\ Chinese Academy of Sciences, Beijing, China \\ ${ }^{2}$ University of Chinese Academy of Science, Beijing, China \\ ${ }^{3}$ Haystack Observatory, Massachusetts Institute of Technology, Westford, Massachusetts, Boston, USA \\ ${ }^{4}$ Institute of Theoretical Physics, Northeast Normal University, Changchun, China \\ Email: shchen@nenu.edu.cn
}

Received December 25, 2013; revised January 20, 2014; accepted January 28, 2014

Copyright (c) 2014 Ziwei Chen, Shi Hao Chen. This is an open access article distributed under the Creative Commons Attribution License, which permits unrestricted use, distribution, and reproduction in any medium, provided the original work is properly cited. In accordance of the Creative Commons Attribution License all Copyrights (C) 2014 are reserved for SCIRP and the owner of the intellectual property Ziwei Chen, Shi Hao Chen. All Copyright (C) 2014 are guarded by law and by SCIRP as a guardian.

\begin{abstract}
Experiments described in this paper show that there is the photoconductive effect of liquid, i.e. when light shines into a sort of alkali, acid or salt solution, the conductivity of the solution will increase. The mechanism of the effect is explained as follows. When hydrated ions in the solution absorb photons with their high enough energies, they will decompose to naked ions and water-molecules. The naked ions can reach an anode or a cathode more easily and faster than the hydrated ions; It is possible that when a molecule in the solution absorbs a photon with its high enough energy, it will decompose to negative and positive ions. Based on the effect, a device producing hydrogen by the solar-energy had been devised.
\end{abstract}

\section{KEYWORDS}

\section{Photoconductive Effect; Solar Energy; Hydrated Ions; Conductivity of Solutions; Hydrogen}

\section{Introduction}

The conventional photoconductive effect is that the conductance of a semiconductor will change when light shines onto the semiconductor. It is one of the photoelectric effects. This effect has been researched in detail [1].

A photoconductive effect of liquid is discussed in the present paper. This effect is different from the photoconductive effect of semiconductor and the Becquerel effect [2]. The photoconductive and photovoltaic effects of semiconductors occur all in semiconductors (solid objects), and go with change of states of electrons in semiconductors. The Becquerel effect is that a potential difference appears between two electrodes in electrolyte when light shines onto the electrode.

The photoconductive effect of liquid is that the conductance of a solution will increase when light shines into the solution (a sort of liquid), but does not shine onto an electrode. In contrast with the Becquerel effect, when light shines into a solution, there is no potential differ- ence appearing in spite of the conductance of the solution increases. The essence of the effect is that one property (conductance) of solutions changes (increases) because light shines into the solutions. It is unrelated to whether there are electrodes in the solutions.

Its mechanism is that a hydrated ion in the solution will be decomposed into one naked ion and water molecules when it absorbs photons or temperature rises. The naked ions can reach more easily and faster an anode or a cathode than the hydrated ions so that the conductance of the solution increases. When a molecule in the solution absorbs a photon with its high enough energy, it will decompose to negative and positive ions which can increase conductance of the solution. It is seen that its mechanism is different from the photoconductive effect of semiconductors and the Becquerel effect.

Based on the effect, a device producing hydrogen, electricity and heating water by the solar-energy had been devised. The utilizing efficiency of the solar energy by the device is higher than $25 \%$. 
In Section 2, the experiments of the photoconductive effect of the potassium hydroxide solution are described; In Section 3, the mechanism of the photoconductive effect of liquid is explained; In Section 4, based on the effect, a device producing hydrogen, electricity and heating water by the solar-energy had been proposed; Section 5 is the conclusions.

\section{An Experiment for the Photoconductive Effect of Liquid}

\subsection{Photoconductive Effect of the $\mathrm{KOH}$ Solution}

Experiment device: one constant-intensity light source (a lamp, sodium-light, GP20Na-B), one direct-current electric source (JWY-30-2A), one voltage meter (C21-V, 0.5 Level) , one electrical current meter (C21-mA, 0.5 Level, Range: $25 \mathrm{~mA}$, Full lattices: 150), one thermometer (WNG-02, Gradation $0.2^{\circ} \mathrm{C}$ ), one square box without top whose cabbage is $4 \times 4 \times 9.6 \mathrm{~cm}^{3}$, $\mathrm{KOH}$, water and carbon-fibre lines. The light source is fixed supra the box and the distance is $4 \mathrm{~cm}$. When the distance to the light source is $3 \mathrm{~cm}$ the spectral characteristics and intensity of the light source is shown in Figure 1.

Experiment process: Two carbon-fibre lines are fixed on the two surfaces inside the box, respectively. The distance between the two carbon-fibre lines is $3.5 \mathrm{~cm}$. Fill $50 \mathrm{~g}$ of potassium hydroxide and $150 \mathrm{~g}$ of water into the box, connect the electrical current meter and the box by the carbon-fibre lines in series. Then make the voltage meter, the current meter and the box in parallel connection, and at last, connect them to the direct-current electrical source. Measure the temperature of the $\mathrm{KOH}$ solution by the thermometer.

Adjust the voltage of the direct-current source to make the current to be $9.8 \mathrm{~mA}$ when the lamp is switched off. The voltage and the light source do not change in the whole experimental process. With add water, keeping constant concentration of the solution by add water.

In this case, the current does not significantly change, and the temperature of the solution increases $0.5^{\circ} \mathrm{C}$ for one hour.

Then open or switch off the lamp. Observe the change of the electric current and the temperature of the $\mathrm{KOH}$ solution every 5 minutes when the lamp is opened or shut. The lamp was opened for 125 minutes, then was switched off for 25 minutes for the first time of the experiment. Measure once every 5 minutes. The number of measurement is 30 times. After 4 hours, the second time of the experiment began. The lamp was opened for 225 minutes, then the lamp was switched off for 75 minutes. Measure once every 5 minutes. The number of measurement is 60 times.

Estimate of the uncertainty. The properties of the electrical current meter are that the level is 0:5 the range is

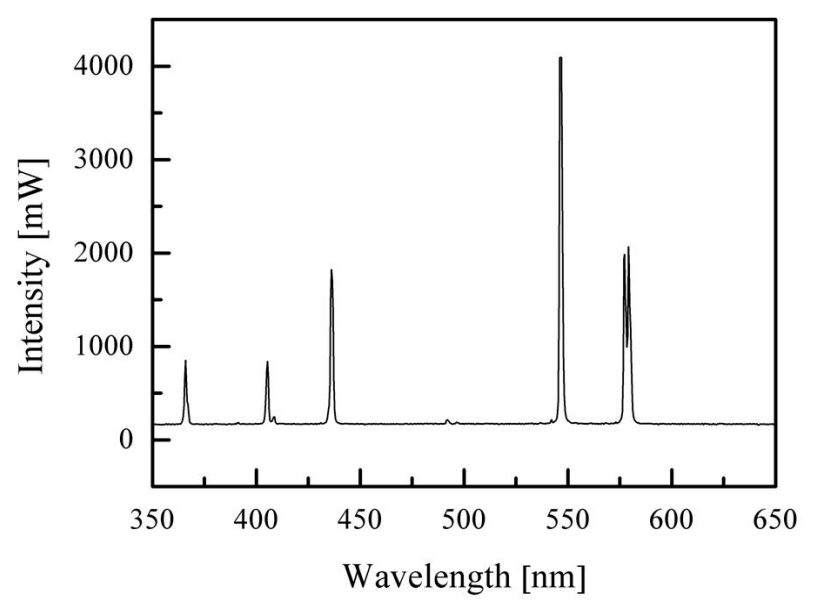

Figure 1. The spectral characteristics and intensity of the light source.

$25 \mathrm{~mA}$ and the number of the full lattices is 150. The number of measurement of current is only one at every time of observation. Hence the accuracy $\Delta E$ of the current meter and the uncertainty $U_{E j}$ of the current can be evaluated as follows.

$$
\begin{aligned}
& \Delta_{E}=0.5 \% \times 25=0.125 \mathrm{~mA}, \\
& u_{E j}=0.125 / \sqrt{3} \cong 0.1 \mathrm{~mA},
\end{aligned}
$$

The properties of the thermometer are that the gradation is $0.2^{\circ} \mathrm{C}$ and the range is $0^{\circ} \mathrm{C}-50^{\circ} \mathrm{C}$. The number of measurement of temperature is only one at every time of observation. Hence the accuracy $\Delta_{T}$ and the uncertainty $\mu_{T j}$ of the temperature are

$$
\Delta_{T}=u_{T j}=0.2^{\circ} \mathrm{C} \text {. }
$$

The change of the electric current and the temperature are shown by Table 1 and Figure 2 for the first time of the experiment. The change of the electric current and the temperature are shown by Table 2 and Figure 3 for the second time of the experiment.

From Table 1 we see that

$$
\begin{gathered}
I(t=25 \times 5)=15.0=1.52 \cdot I(t=0) ; \\
T(t=25 \times 5)=25.0=1.45 \cdot T(t=0) . \\
I(t=19 \times 5)=14.3=1.19 \cdot I(t=28 \times 5) . \\
T(t=19 \times 5)=24.0=T(t=28 \times 5) .
\end{gathered}
$$

It is seen from (1)-(2) that the current and the conductance increase when light shines into the solution. It is seen from (2)-(3) that the temperature and voltage when $t=19 \times 5$ are the same as those when $t=28 \times 5$, but the current when $t=19 \times 5$ is different from that when $t=28 \times 5$, Hence the current is not determined by only temperature and voltage, but is related to the light.

From Table 2 we see that 
Table 1. Change of the electric current and the temperature for the first time of the experiment. In table 1, L, t, I, T, S, 0 and F denote "lamp", “time”, “current”, “temperature", "shuto", “open” and "-nally", respectively. The time interval is $5 \mathrm{~m}$ inites. The unit of the current is "Ma". "Finally" implies that the current and the temperature are observed after a two hours.

\begin{tabular}{cccccccccccc}
\hline $\mathrm{L}$ & $\mathrm{S}$ & 0 & 0 & 0 & 0 & 0 & 0 & 0 & 0 & 0 & 0 \\
$\mathrm{t}$ & 0 & 1 & 2 & 3 & 4 & 5 & 6 & 7 & 8 & 9 & 10 \\
$\mathrm{I}$ & $9: 8(1)$ & $9: 9(1)$ & $10: 8(1)$ & $11: 8(1)$ & $12: 0(1)$ & $12: 2(1)$ & $12: 4(1)$ & $12: 5(1)$ & $12: 6(1)$ & $12: 8(1)$ & $12: 9(1)$ \\
$\mathrm{T}$ & $17: 3(2)$ & $17: 3(2)$ & $17: 3(2)$ & $18: 0(2)$ & $18: 0(2)$ & $18: 5(2)$ & $19: 0(2)$ & $19: 5(2)$ & $20: 0(2)$ & $20: 4(2)$ & $21: 0(2)$ \\
$\mathrm{L}$ & 0 & 0 & 0 & 0 & 0 & 0 & 0 & 0 & 0 & 0 & 0 \\
$\mathrm{t}$ & 11 & 12 & 13 & 14 & 15 & 16 & 17 & 18 & 19 & 20 & 21 \\
$\mathrm{I}$ & $13: 1(1)$ & $13: 4(1)$ & $13: 5(1)$ & $13: 7(1)$ & $13: 7(1)$ & $14: 0(1)$ & $14: 00(1)$ & $14: 2(1)$ & $14: 3(1)$ & $14: 5(1)$ & $14: 6(1)$ \\
$\mathrm{T}$ & $21: 2(2)$ & $22: 0(2)$ & $22: 0(2)$ & $22: 52(2)$ & $23: 0(2)$ & $23: 1(2)$ & $23: 5(2)$ & $24: 0(2)$ & $24: 0(2)$ & $24: 1(2)$ & $24: 3(2)$ \\
$\mathrm{L}$ & 0 & 0 & 0 & 0 & $\mathrm{~S}$ & $\mathrm{~S}$ & $\mathrm{~S}$ & $\mathrm{~S}$ & $\mathrm{~S}$ & $\mathrm{~S}$ & \\
$\mathrm{t}$ & 22 & 23 & 24 & 25 & 26 & 27 & 28 & 29 & 30 & $\mathrm{~F}$ & \\
$\mathrm{I}$ & $14: 7(1)$ & $14: 9(1)$ & $15: 0(1)$ & $15: 0(1)$ & $13: 3(1)$ & $12: 3(1)$ & $12: 0(1)$ & $11: 7(1)$ & $11: 6(1)$ & $8: 7(1)$ \\
$\mathrm{T}$ & $24: 8(2)$ & $25: 0(2)$ & $25: 0(2)$ & $25: 0(2)$ & $25: 0(2)$ & $24: 5(2)$ & $24: 0(2)$ & $23: 5(2)$ & $23: 0(2)$ & $15: 0(2)$ \\
\hline
\end{tabular}

Table 2. Change of the electric current and the temperature for the second time of the experiment.

\begin{tabular}{|c|c|c|c|c|c|c|c|c|c|c|c|}
\hline $\mathrm{L}$ & $S$ & 0 & 0 & 0 & 0 & 0 & 0 & 0 & 0 & 0 & 0 \\
\hline $\mathrm{t}$ & 0 & 1 & 2 & 3 & 4 & 5 & 6 & 7 & 8 & 9 & 10 \\
\hline I & $8: 7(1)$ & $9: 9(1)$ & $10: 7(1)$ & $10: 8(1)$ & $10: 8(1)$ & $10: 8(1)$ & $11: 1(1)$ & $11: 4(1)$ & $11: 5(1)$ & $11: 7(1)$ & $11: 9(1)$ \\
\hline $\mathrm{T}$ & $15: 0(2)$ & $15: 0(2)$ & $15: 4(2)$ & $16: 0(2)$ & $16: 8(2)$ & $17: 2(2)$ & $18: 0(2)$ & 19:0(2) & $19: 4(2)$ & $20: 0(2)$ & $20: 5(2)$ \\
\hline $\mathrm{L}$ & 0 & 0 & 0 & 0 & 0 & 0 & 0 & 0 & 0 & 0 & 0 \\
\hline $\mathrm{t}$ & 11 & 12 & 13 & 14 & 15 & 16 & 17 & 18 & 19 & 20 & 21 \\
\hline I & $12: 1(1)$ & $12: 1(1)$ & 12:2(1) & $12: 4(1)$ & $12: 6(1)$ & $12: 7(1)$ & $12: 8(1)$ & 13:0(1) & 13:1(1) & $13: 2(1)$ & $13: 3(1)$ \\
\hline $\mathrm{T}$ & $21: 0(2)$ & 21:1(2) & 22:0(2) & $22: 0(2)$ & $22: 6(2)$ & 23:0(2) & $23: 2(2)$ & $23: 2(2)$ & $24: 0(2)$ & $24: 2(2)$ & $24: 5(2)$ \\
\hline $\mathrm{L}$ & 0 & 0 & 0 & 0 & 0 & 0 & 0 & 0 & 0 & 0 & 0 \\
\hline $\mathrm{t}$ & 22 & 23 & 24 & 25 & 26 & 27 & 28 & 29 & 30 & 31 & 32 \\
\hline I & $13: 4(1)$ & 13:6(1) & 13:7(1) & 13:8(1) & 13:9(1) & $14: 0(1)$ & $14: 1(1)$ & $14: 2(1)$ & $14: 3(1)$ & $14: 4(1)$ & $14: 4$ \\
\hline $\mathrm{T}$ & $24: 5(2)$ & $25: 0(2)$ & $25: 3(2)$ & $25: 5(2)$ & $25: 9(2)$ & $26: 0(2)$ & $26: 1(2)$ & $26: 2(2)$ & $26: 5(2)$ & $26: 5(2)$ & $26: 7$ \\
\hline $\mathrm{L}$ & 0 & 0 & 0 & 0 & 0 & 0 & 0 & 0 & 0 & 0 & 0 \\
\hline $\mathrm{t}$ & 33 & 34 & 35 & 36 & 37 & 38 & 39 & 40 & 41 & 42 & 43 \\
\hline I & $14: 8(1)$ & $14: 8(1)$ & $15: 0(1)$ & $15: 1(1)$ & $15: 2(1)$ & $15: 2(1)$ & $15: 3(1)$ & $15: 3(1)$ & $15: 3(1)$ & $15: 4(1)$ & $15: 3(1)$ \\
\hline $\mathrm{T}$ & $27: 0(2)$ & $27: 1(2)$ & $27: 2(2)$ & $27: 3(2)$ & $27: 4(2)$ & $27: 4(2)$ & $27: 5(2)$ & 28:0(2) & 28:0(2) & 28:0(2) & $28: 2(2)$ \\
\hline $\mathrm{L}$ & 0 & 0 & 0 & 0 & S & $\mathrm{S}$ & $\mathrm{S}$ & S & S & S & S \\
\hline $\mathrm{t}$ & 44 & 45 & 46 & 47 & 48 & 49 & 50 & 51 & 52 & 53 & 54 \\
\hline I & $15: 3(1)$ & $15: 3(1)$ & 13:3(1) & $12: 6(1)$ & $12: 0(1)$ & $11: 6(1)$ & $11: 0(1)$ & $10: 7(1)$ & $10: 4(1)$ & $10: 1(1)$ & $10: 0(1)$ \\
\hline $\mathrm{T}$ & $28: 3(2)$ & $28: 4(2)$ & 28:0(2) & $27: 1(2)$ & $26: 8(2)$ & $26: 0(2)$ & $25: 0(2)$ & $245(2)$ & 24:0(2) & $23: 5(2)$ & $23: 0(2)$ \\
\hline $\mathrm{L}$ & $\mathrm{S}$ & $\mathrm{S}$ & $\mathrm{S}$ & $\mathrm{S}$ & $\mathrm{S}$ & $\mathrm{S}$ & $\mathrm{S}$ & & & & \\
\hline $\mathrm{t}$ & 55 & 56 & 57 & 58 & 59 & 60 & F & & & & \\
\hline I & $9: 8(1)$ & $9: 6(1)$ & $9: 5(1)$ & $9: 3(1)$ & $9: 2(1)$ & $9: 1(1)$ & $7: 6(1)$ & & & & \\
\hline $\mathrm{T}$ & $22: 6(2)$ & $22: 3(2)$ & 22:0(2) & 21:5(2) & 21:4(2) & 21:0(2) & $17: 0(2)$ & & & & \\
\hline
\end{tabular}

$$
\begin{gathered}
I(t=45 \times 5)=14.8=1.76 \cdot I(t=0), \\
T(t=45 \times 5)=28.4=1.89 \cdot T(t=0) . \\
I(t=34 \times 5)=14.8=1.17 \cdot I(t=47 \times 5), \\
T(t=34 \times 5)=27.1=T(t=47 \times 5) .
\end{gathered}
$$

Here $I(t=25 \times 5)$ and $I(t=45 \times 5)$ are the stable currents.

From Tables 1 and 2, Figures 2 and 3 and (1)-(2) and (4)-(5) the following results can been obtained.

1) The electric current is significantly higher when light shines into the solution than that when no light shines into the solution;

2) When two temperatures are the same, the electric current is significantly higher when the light shines the 


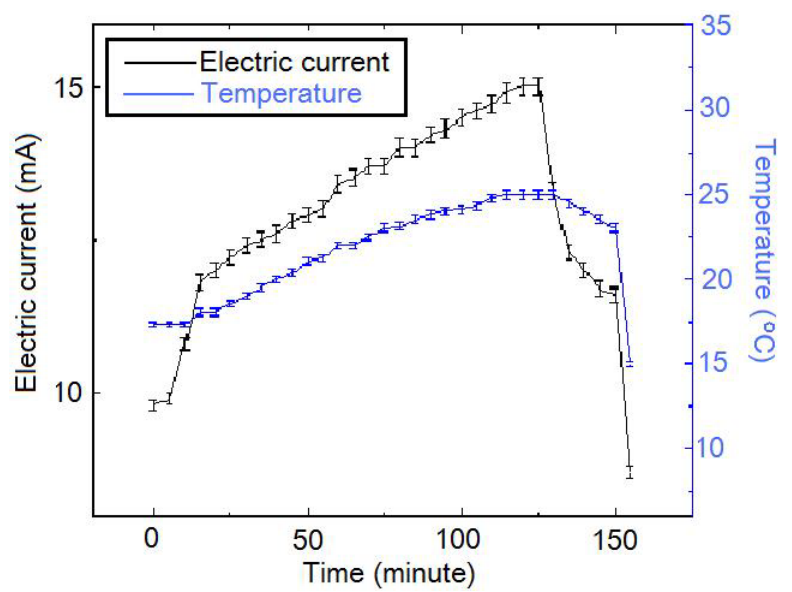

Figure 2. Change of the electric current and the temperature for the first time of the experiment.

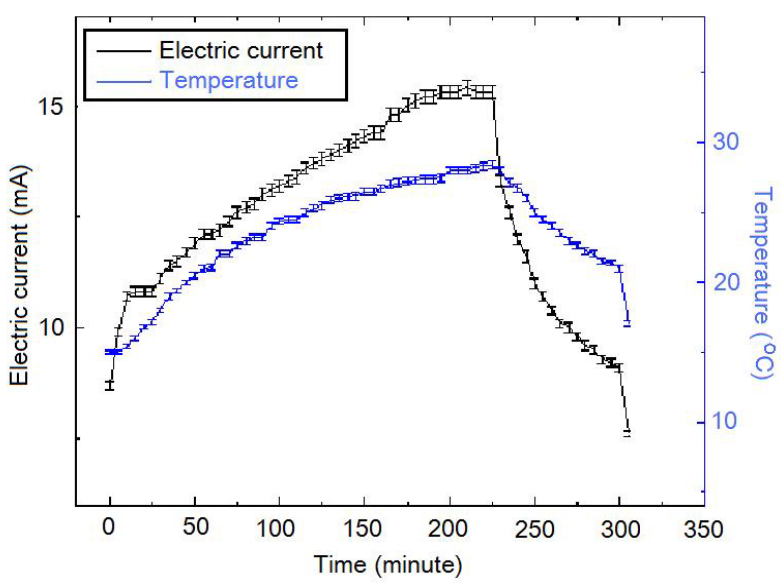

Figure 3. Change of the electric current and the temperature for the second time of the experiment.

solution than that when no light shines the solution;

3) There is the maximum of the electric current $I_{\max }$ so that when $I=I_{\max }$, the electric current no longer increases, although light continues to shines into the solution.

It is seen that the electric current is not determined by only voltage, and is not determined by only temperature and voltage as well, and the electric conductivity of the solution has an obvious increase when light shines into the solution. This is the photoconductive effect of liquid. Photoconductive effects of the $\mathrm{HCl}$ vor the $\mathrm{NaCl}$ solution.

Replacing $\mathrm{KOH}$ by $\mathrm{HCl}$ or $\mathrm{NaCl}$, we had accomplished the experiments and had obtained the results analogous to the $\mathrm{KOH}$ experiment. The main difference from the $\mathrm{KOH}$ experiment is that $\mathrm{Cl}_{2}$ going from the solution of $\mathrm{HCl}$ or $\mathrm{NaCl}$ solution should be sent back the primary solution to preserve the concentration of the solutions. We will explain the two experiments in other paper in detail.

\subsection{Mechanism of the Photoconductive Effect of Liquid}

1) A hydrated ion in a solution will be decomposed into one naked ion and water molecules when it absorbs photons with their energies to be high enough or the temperature of the solution to be high enough.

The $\mathrm{K}^{+}$or $\mathrm{OH}^{-}$ions in the solution are not existent solely, but combine with water molecules and form into hydrated potassium ions and hydrated hydroxide ions, respectively. The number of water-molecules combined by an ion is changeable as temperature and the intensity and frequency of the light shining the solution. The number is fewer when temperature or the intensity of the light is larger. When the temperature or the intensity of light is high enough, the ions no longer combine with water molecules.

Let there be $n_{K}(T)$ water molecules in a hydrated potassium ion when temperature is $T$. The energies of $n_{K}(T)$ photons to disassemble the $n_{K}(T)$ watermolecules from the hydrated ion are different from each other. The energy $E_{K}(T)$ disassembling the last water molecule from the hydrated ion is the largest. When a hydrated potassium ion absorbs $n_{K}(T)$ photons with the energy $E \geq E_{K}(T)$, the hydrated potassium ion will become a naked ion and water molecules.

It can be seen from the experiment mentioned above that a hydrated $\mathrm{K}^{+}$ion in the $\mathrm{KOH}$ solution will lose a water molecule when it absorbs a photon with its energy $h c / \lambda_{0}=E_{0}$, here $\lambda_{0}=550 \mathrm{~nm}$ in Figure 1. In fact, $E_{K}<<E_{0}$. The boiling point of the $\mathrm{KOH}$ solution with its concentration $50 /(50+150)=25 \%$. Is $118^{\circ} \mathrm{C}=T_{K B}$.

This implies that a hydrated $\mathrm{K}^{+}$ion can lose one water molecule when it absorbs one photon with its energy

$$
E_{K}=k T_{K B}=8.6 \times(273+118)=0.034 \mathrm{eV} \equiv E_{B 0}, .
$$

here $k$ is the Boltzmann constant, and $E_{K}$ is the energy of a Mid-infrared photon with its wavelength $\lambda_{K}=36 \mu \mathrm{m}$. Let $E_{B T}$ and $E_{T}$ be the total energy of such photons with its energy $E_{B} \geq E_{B 0}$ in the solar light and the total energy of the solar energy, respectively, $E_{B T} / E_{T} \geq 90 \%$. This implies that the part of the sunlight can cause hydrated the $\mathrm{K}^{+}$ions to loss their water molecules. Thus, the coefficient absorbing solar energy by the hydrated $\mathrm{K}^{+}$ ions is larger.

There is the result analogous to the hydrated $\mathrm{K}^{+}$ions for the hydrated $\mathrm{OH}^{-}$ions in the solution.

The naked ions can arrive at the anode or the cathode more easily and faster than the hydrated ions in the same electric field. Hence the conductance and the intensity of current of the solution will increase. 
2) Water-molecules and other molecules in the solution can be decomposed into positive and negative ions as well, provided the molecules absorb photons with large enough energy. For example, a $\mathrm{H}_{2} \mathrm{O}$ molecule can be decomposed into $\mathrm{H}^{+}$and $\mathrm{OH}^{-}$when it absorbs a photon with the energy $E>E_{W}$, here $E_{W}=5.19 \mathrm{eV}$ is the energy disassembling $\mathrm{H}_{2} \mathrm{O}$ into $\mathrm{H}^{+}$and $\mathrm{OH}^{-}$. when the atmospheric pressure $P=101.3 \mathrm{KPa}$ and temperature $T=293 K$. The wavelength corresponding to $E_{W}$ is $\lambda_{W}=239 \mathrm{~nm} . E_{W}$ can decrease because of interactions of ions or some catalysts or increase of temperature. The ions can increase the conductance of the solution as well.

3) Let the numbers of the naked $\mathrm{K}^{+}, \mathrm{OH}^{-}$and $\mathrm{H}^{+}$ions be $n\left(\mathrm{~K}^{+}\right), n\left(\mathrm{OH}^{-}\right)$and $\mathrm{n}\left(\mathrm{H}^{+}\right)$, respectively, there must be

$$
n\left(\mathrm{OH}^{-}\right)=n\left(\mathrm{~K}^{+}\right)+n\left(\mathrm{H}^{+}\right), \quad n\left(\mathrm{~K}^{+}\right)>>n\left(\mathrm{H}^{+}\right) .
$$

This is because $E_{K} \sim E_{\mathrm{OH}}<<E_{W}$. Thus, the ions arriving at the cathode are mainly the naked $\mathrm{K}^{+}$ions, and the ions arriving at the anode are the naked $\mathrm{OH}^{-}$ions. There are the following reactions.

$$
\begin{gathered}
\mathrm{K}^{+}+\mathrm{e}=\mathrm{K}, \\
2 \mathrm{~K}+2 \mathrm{H}_{2} \mathrm{O}=2 \mathrm{KOH}+\mathrm{H}_{2} \uparrow, \\
4\left(\mathrm{OH}^{-}-\mathrm{e}\right)=2 \mathrm{H}_{2} \mathrm{O}+\mathrm{O}_{2} \uparrow .
\end{gathered}
$$

In the process, the quantity of $\mathrm{K}^{+}$ions in the solution does not change, while the quantity of water molecules in the solution decreases. The result is the same as the process of electrolyzing water directly. But the essence of he process is different from that of electrolyzing water directly. In contrast with the process of electrolyzing water, the naked $\mathrm{K}^{+}$ions selves get charges at the cathode and becomes $\mathrm{K}^{+}$atoms. A potassium atom $\mathrm{K}$ replaces a hydrogen atom $\$ H \$ \backslash$ in a water molecule, and then hydrogen gas is release from the solution. The hydroxide ions losing electrons at the anode join each other and form water molecules and oxygen gas.

In the process, the energy decomposing water comes from light energy. The hydrated ions are disassembled into naked ions and water molecules due to absorption of photons. Besides, the kinetic energy of the naked ions will increase because of interaction of electromagnetic wave of low frequency so that the temperature of the solution increases. The naked ions can get energy from a photon with its high energy by the Compton scattering as well. It is seen that the solutions containing ions can absorb more light energy than solar panels, because $90 \%$ of the solar light satisfies the condition

$h c / \lambda \geq E_{K}=0.034 \mathrm{eV}$ and such light can be absorbed by the solution.

4) When temperature or the intensity of light is high enough, all hydrated ions will be disassembled into na- ked ions and water-molecules. Consequently, the number of ions in the solution cannot continue to increase. In fact, in this case, it is possible that the conductance will decrease when temperature continues to increase. Thus, there must be the maximum of the electric current $I_{\max }$, i.e. the electric current no longer increases when $I-I_{\max }$, although light continues to shine into the solution.

Comparing the current $I(t=0)=9.85 \mathrm{~mA}$ before the light-source is opened and the current $I(t=F)=8.70 \mathrm{~mA}$ after the light source is shut, we find $I(t=0)>I(t=F)$. This is because some ions with opposite charges have integrated into new molecules in the process.

5) There are carbon particles and compounds of carbon in the solution because the two electrodes are composed of carbon fibre. The carbon particles and compounds of carbon can combine with the naked ions and influence the conductance of the solution. When some particles, e.g. $\mathrm{ZnO}, \mathrm{Zn}(\mathrm{OH})_{2}, \mathrm{~K}_{2}\left[\mathrm{Zn}(\mathrm{OH})_{4}\right]$ or $\mathrm{TiO}_{2}$ etc., are added into the solution, the conductance of the solution can be influenced. The photoconductive effect of the solution should be regarded as a result of the synthetically interaction of all components in the solution.

6) Supposing the conductance of a solution is $G_{0}=1 / R_{0}$ when the voltage between two electrodes is $V=V_{0}$ and there is no light to shine into the solution, and the conductance is $G_{1}=n G_{0}$ when $V_{1}=V_{0}$ and light shines into the solution, we have

$$
I_{1}=V_{1} G_{1}=n I_{0}, \quad P_{1}=I_{1}^{2} / G_{1}=n P_{0} .
$$

Here we consider only the conductance of the solution for simplicity. If there is no light to shine the solution, in order to get the equal quantity of hydrogen, the current must be the same as $I_{1}$. In the case, the voltage and power are respectively

$$
V_{2}=I_{1} / G_{0}=n V_{0}, \quad P_{2}=I_{1}^{2} / G_{0}=n P_{1} .
$$

There must be $n>1$ due to the photoconductive effect of liquid. We see from Tables 1 and 2 that $n=15 / 9.85=1.52$ and $15.3 / 8.7=1.76$ in the present experiment. It is seen that expending electric energy when light shines into the solution is less than that when there is no light to shine the solution when the same quantity of hydrogen is produced, i.e. $P_{2}>P_{1}$. This implies that the light energy transforms into the hydrogen energy.

Sum up, when hydrated ions in the solution absorb photons, the hydrated ions will decompose to naked ions and water-molecules. The naked ions can arrive more easily and faster at an anode or a cathode than the hydrated ions so that the conductance increases. Because of the interactions of ions or some catalysts in the solution, $E_{W}(T)$ can decrease, i.e. the water-molecules in the 
solution are decomposed more easily. When a molecule in the solution absorbs a photon with its large enough energy, it will decompose to negative and positive ions which can increase conductance of the solution. Besides, the solutions containing ions can absorb more light energy than solar panels.

\section{An Application of the Photoconductive Effect of Liquid}

The photoconductive effect of liquid could be used in many aspects. For example, based on the effect, we can use solar energy to produce more hydrogen and oxygen. Suppose the rate of generating hydrogen is proportional to the electric current. When the solar light shines into the solution, the conductance and electrical current of the solution will increase. Consequently, the quantity of generating hydrogen will increase. As mentioned above, expending electric energy when light shines into the solution is less than that when there is no light to shine the solution when the same quantity of hydrogen is produced.

As mentioned above, $90 \%$ of the wave lengths of the solar light satisfies the condition $h c / \lambda>E_{K} \approx 0.034 \mathrm{eV}$ and such light can be absorbed by the hydrated $\mathrm{K}^{+}$ions. Hence the hydrated ions in the $\mathrm{KOH}$ solution are easily disassembled into naked ions and water molecules when the solution is shone by the solar light. Water can dissolve many substances so that electromagnetic waves of many wave lengths can be absorbed by the water solutions. Thus, the absorption coefficient of the solar energy by a water solution could be high.

Based on the photoconductive effect of liquid, a device producing hydrogen and heating water by the solar-energy had been devised (see details in Ziwei Chen's related patents). The utilizing efficiency of the solar energy by the device is higher than $25 \%$.

\section{Conclusions}

The experiment described shows that the conductance of the $\mathrm{KOH}$ solution will increase when light shines into the solution but does not shine onto an electrode, i.e. there is the photoconductive effect of liquid. The origin of this effect is that the hydrated ions will decompose to naked ions and water molecules when hydrated ions absorb photons with large enough energy. The naked ions can reach electrodes more easily and faster than the hydrated ions so that the conductance of the solution increases. When a molecule in the solution absorbs a photon with large enough energy, the molecule will decompose to negative and positive ions. Thus, the conductance of solutions can increase because light shines into the solutions. In contrast with the Becquerel effect, when light shines into a solution, there is no potential difference appearing in spite of the conductance of the solution increases. The essence of the effect is that one property (conductance) of solutions changes (increases) because light shines into the solutions. It is unrelated to whether there are electrodes in the solutions.

Based on the effect, a device producing hydrogen by the solar-energy had been devised. The device can improve the efficiency of solar energy utilization.

It can be seen that the effect exists in other liquid.

\section{Acknowledgements}

The work is supported by tau-charm physics research $\mathrm{KJ}(\mathrm{x} 2-\mathrm{yw}-\mathrm{N} 29)$.

\section{REFERENCES}

[1] R. H. Bube, "Photoconductivity of Solids," New York, 1960, pp. 56-74,325-356.

[2] E. D. Korlyakov, “The Becquerel Effect," Soviet Physics Journal, Vol. 12, No. 8, 1968, pp. 1054-1056. 